\title{
On the Role of Topology in Focus+Context Visualization
}

\author{
Ivan Viola and Meister Eduard Gröller \\ Department of Informatics, University of Bergen, Norway \\ Institute of Computer Graphics and Algorithms, \\ Vienna University of Technology, Austria \\ ivan@ii.uib.no, meister@cg.tuwien.ac.at
}

In this paper three types of visualization scenarios are discussed, where topology improves the readability of particular visualization results. The first type combines topology information represented by simple graphical primitives with other forms of visual representations. The second type uses the topology information to define the relevance of objects within the data. The relevance is reflected in the visualization by applying the cut-away concept. The third type of visualizations is based on the change of topology of the underlying data to increase visibility of the most interesting information. Every type handles topology in a different way. This illustrates various roles of topology in scientific visualization.

\section{Introduction}

Three-dimensional visualization is becoming an essential tool for the analysis of various scientific problems. A major problem are the very large data sizes that are hard to handle. For example the rapid development of high-precision medical imaging modalities causes the amount of data to steadily increase. Due to the increasing power of modern CPUs, mathematical simulations of scientific phenomena deliver huge amounts of result data. Processing and visualization of time-varying data is becoming practicable in many applications. The added temporal dimension, furthermore increases the data sizes considerably.

Large data sizes entail two fundamental problems: The first is data manipulation with respect to data enhancement and processing in general. In this paper we focus on the second problem, which is the appropriate visual representation of the underlying data. The amount of relevant information is often relatively small as compared to the overall amount of acquired data. Therefore these small, interesting features have to be emphasized visually. 


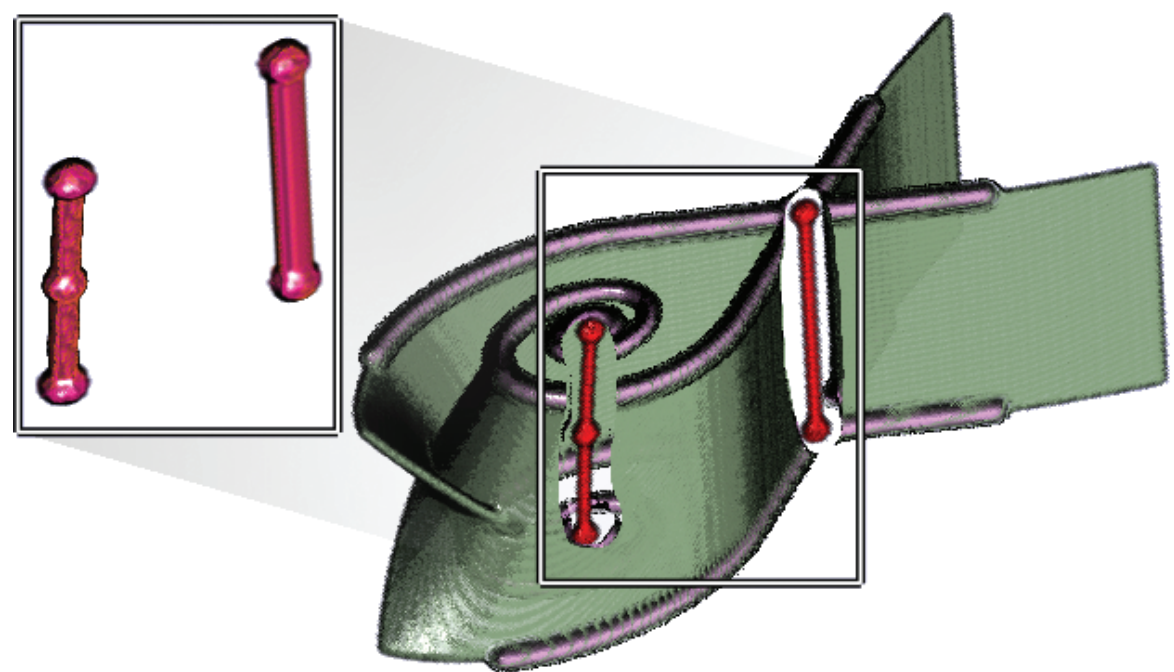

Fig. 1. Periodic Blue Sky Bifurcation: The topology can be clearly depicted by simple and sparse geometry. Critical lines occluded by separation surfaces are unveiled by using importance-driven visualization, where importance is defined through topological characteristics. Additional emphasis is achieved by displacement in the spirit of exploded views. The polygonal model is courtesy of Tino Weinkauf and Holger Theisel.

We generalize the term feature as a part of the data that is of special interest to the user. To highlight the role of topology in visualization, we concentrate in our examples on features that have some topological characteristics. Examples are singularities in flow visualization, or cancer lung nodules in medical visualization.

In many cases visualization of the features alone does not clearly illustrate the inspected problem and thus does not satisfy the goal of an expressive visualization. The data surrounding the features includes more information and some of this information is necessary to communicate the visualized problem. Spatial position and vicinity to other structures can be very important for example. A visualization technique that combines the most interesting data (i.e., a feature) with surrounding information of smaller information value is in general denoted as focus+context visualization [2].

Combining the most interesting features with the contextual information has to be done carefully to avoid visual overload. In this paper we discuss several visualization scenarios, where topology helps to effectively convey the information in focus+context visualizations:

- Visualization of topology: An appropriate visual representation is the key to an effective focus+context visualization. The general idea is to provide visualizations as simple as possible to clearly describe the data. Simple spheres, lines, arrows or other basic geometric elements are used 
to describe abstract characteristics of the data such as topology. This abstract visual representation is very sparse, but also very clear and easily understandable. In focus + context visualization such information is combined with denser visual representations that describe the data in more detail. An abstract visual representation depicting the topology is often used in flow visualization or visualization of complex dynamical systems. Specific examples will be discussed in more detail in Section 2.

- Topology-driven visualization: Features within the data can be characterized by various properties, e.g., by their topological characteristics. Such characteristics can be described by very simple geometry, which can be used as an underlying structure that drives the visualization. This structure is used for example in visualization to guarantee the visibility of important features. Specific examples will be given in Section 3.

- Topology changes of the underlying data: The original arrangement of structures in the data is often not optimal to achieve a particular goal through visualization. It is sometimes easier to change the topology of the data in order to clearly see particular features. Examples of changing the topology (e.g., from cylindrical to planar) will be presented in Section 4.

A visualization example illustrating the role of topology in visualization is shown in Figure 1. Here all three scenarios are combined together. The Periodic Blue Sky Bifurcation [7] is represented sparsely by simple geometric elements. The goal is to visualize all topological structures, i.e., critical points, critical lines, and separatrices. The visualization is enhanced by cut-away views and a placement in spirit of exploded views to focus the attention to critical lines and points. After incorporating cut-away views the visual presence of these small features is more balanced with visually more prominent separatrices. Thus the overall visual information is increased.

In the next sections we will explain the relevance of topology in the visualization for specific applications.

\section{Visualization of Topology}

The relevance of features in an image is given through their visual representations, which may vary from an abstract to a more direct visual representation. A direct representation does not use any abstraction and is easy to interpret. However for a large dataset or in higher dimensions it easily produces dense, cluttered images that are visually overloaded. The other end of the spectrum are highly abstract (derived) data representations. They typically produce rather sparse images but can require quite some effort to understand. Showing MRI data of the brain as greyscale images is a rather direct representation. Showing the same data through diffusion tensor imaging is a more abstract representation. Another example of a direct representation are satellite photographs. A map with city icons scaled by population including labels and 


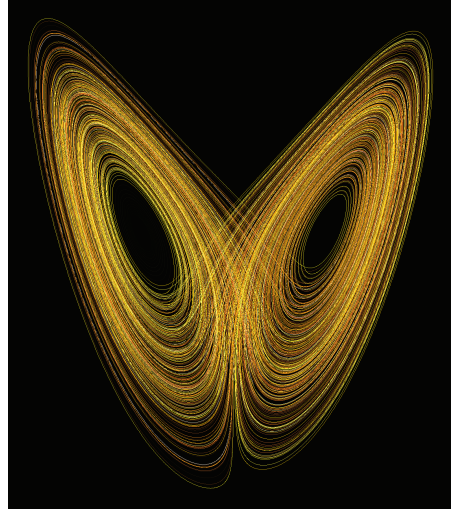

(a)

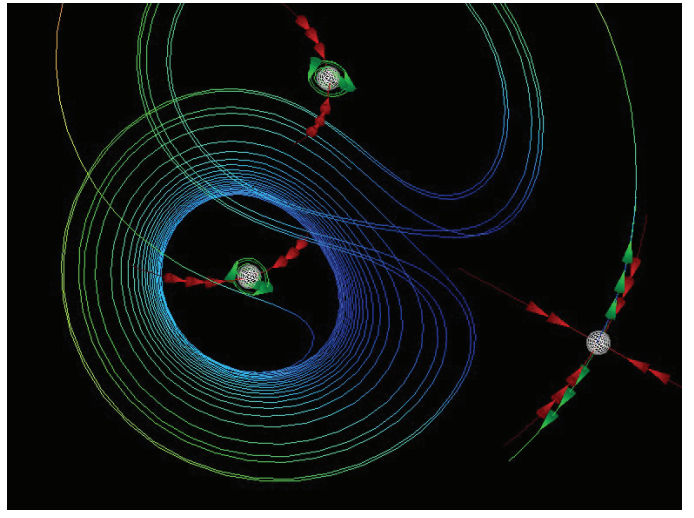

(b)

Fig. 2. Lorenz attractor: (a) direct representation through a streamline and (b) descriptive visualization of the system with emphasis of critical points [4].

connected by transport infrastructure is an abstract representation. There are many ways to design appropriate visual representations. We will focus on those where the visualization of topology significantly increases the visual information content.

The visual encoding of topology information may consist of just a few points, lines, sometimes surfaces. In cases where a direct visual representation would lead to visual clutter, the visualization of topology is a very effective way to show the most important information of the data. There is no visual overload and image space is saved to display further information. In the following we discuss two application domains where the visualization of topology efficiently conveys the characteristics of the underlying data.

\subsection{Visualization of Complex Dynamical Systems}

Topological visual representations are well known from complex dynamical systems. The direct representation of a complex dynamical system can lead to a visual overload. Visualization of topology on the other hand allows to focus the attention to the most relevant features. Here the visual emphasis of the most relevant structures is analogous to the difference between an aerial photograph and a geographic map.

In Figure 2 the Lorenz attractor is represented by (a) a simple streamline and (b) topological information of the underlying dynamical system. The first representation is depicting the overall shape of the system whereas the visualization of topology emphasizes critical points of the system. This information explains the behavior of the dynamical system more effectively. For example 
the alternating dominance of two critical points is caused by the third critical point. This information can by hardly extracted from the streamline plot only.

\subsection{Visualization of Flow}

Flow visualization is another application domain where topological visual representations are effectively used. In some cases features are not clearly defined, i.e., there are no explicit boundaries between features. Interesting features might be vortex cores, fixed points (such as nodes, saddles, vortices), separatrices etc. For a clear visualization of a particular phenomena, the topological information of interesting features is crucial. Figure 3 shows an example of a focus+context visualization of a three-dimensional flow. Here various levels of abstraction in the visual representation are present. The topological information is given by a cycle around the $z$-axis. The flow is directly represented by spot noise [9] on the Poincaré cross section [4]. A streamsurface in orange and a streamline in green provide further abstract context information. The sparse topological information indicates the overall characteristics of the system. The dense and direct depiction of the flow is limited to just a small region of the data to avoid visual clutter. Further examples of topological visual representations of flow can be found in related works $[6,8,13,14]$.

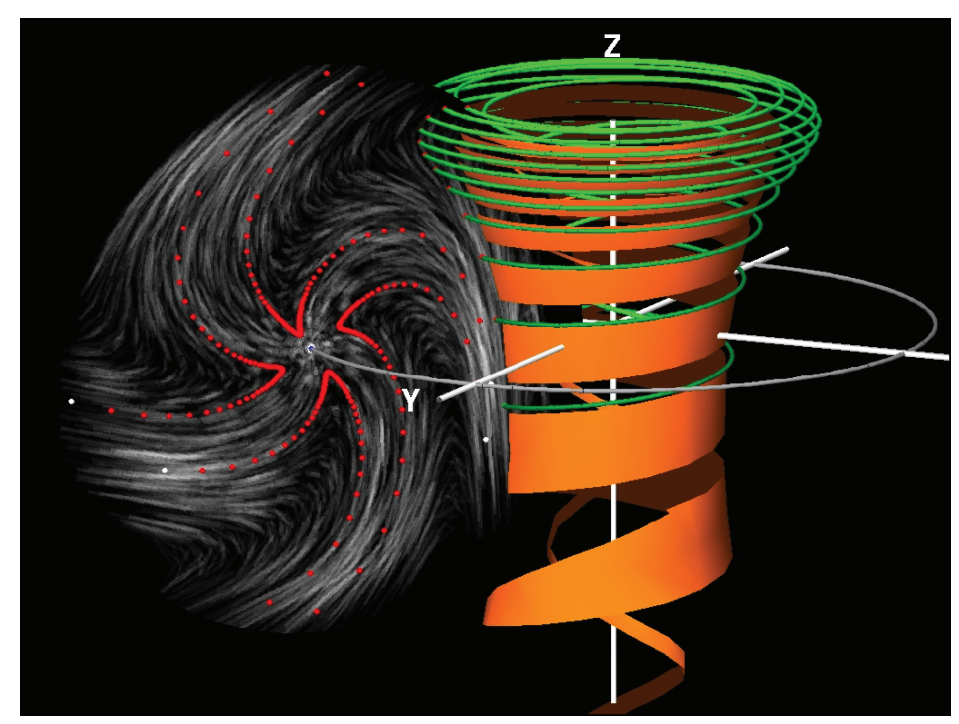

Fig. 3. Focus+context visualization of flow topology using Poincaré sections [4]. 


\section{Topology-Driven Visualization}

The previous section addressed visualizations where topological characteristics increase the visual content despite the fact that this information is represented very sparsely. In this section we will focus on visualization techniques where the topological information drives the visualization algorithm to emphasize interesting objects.

The concept of topology-driven visualization is analogous to importancedriven feature enhancement [12]. The traditional volume visualization pipeline assigns to features optical properties like color and opacity. With importancedriven feature enhancement another dimension is used which describes the importance of interesting objects. In topology-driven visualization the importance is defined through topological characteristics that have the highest priority to be clearly visible. Prior to image synthesis, the visibility of interesting objects is estimated. If less important objects are occluding objects that are more interesting, the less important ones are rendered more sparsely, e.g., more transparently. If the same object does not cause any unwanted occlusions in other regions of the image, it is rendered more densely, e.g., opaque, in order to see its shape more clearly. This allows to see all interesting structures irrespective if they would be occluded or not, and the less important parts are still visible as much as possible. This concept has been inspired by expressive illustration techniques known as cut-aways or ghosted views. We will show their effective usage in the scope of medical and meteorological applications.

\subsection{Visualization of Early-Stage Lung Cancer}

The goal of visualization of early stage lung cancer is to improve the diagnosis and to increase the probability of patient survival. The automatic diagnostic process is divided into two parts. The first part is the detection stage where the data is classified according to the probability of a tumor presence. The second part is the visualization of these suspicious regions. The medical expert is guided by the application to the regions where with high probability lung nodules are present. Thus the time-consuming process of slice-by-slice inspection is replaced by an automatic process. The medical expert finally has to validate which regions are considered as nodules and which regions are false positives.

Nodules are small features in human lungs with a spherical shape. Healthy lungs feature only tubular structures of two categories: airways and blood vessels. Healthy lungs can be considered of genus zero. Each lung nodule is seen as a topological hole in the healthy lungs. The presence of a nodule changes the topology of the lungs and the number of nodules defines the genus.

The detection part is based on local shape properties of a sample position. The detection algorithm is in spirit of the method proposed by Sato et al. [5]. Local shape properties are derived from the Hessian matrix which consists of the second-order partial derivatives [3]. The eigenvalues of the Hessian 
matrix are used to determine the probability of a spherical shape (nodule). The nodule shape is identified due to its different shape characteristics as compared to other tissues within the human lungs.

The visualization part is based on importance-driven feature enhancement. Here the feature is a part of the volume that has a high probability of being a nodule, i.e., a topological hole in the healthy tissue. Volume regions that occlude these suspicious areas are represented sparsely. Visualizations show the spatial position of the most interesting regions irrespective from the viewpoint settings. This increases the expressivity as compared to traditional visualization methods (e.g., direct volume rendering or slicing). Result visualizations are shown in Figure 4.
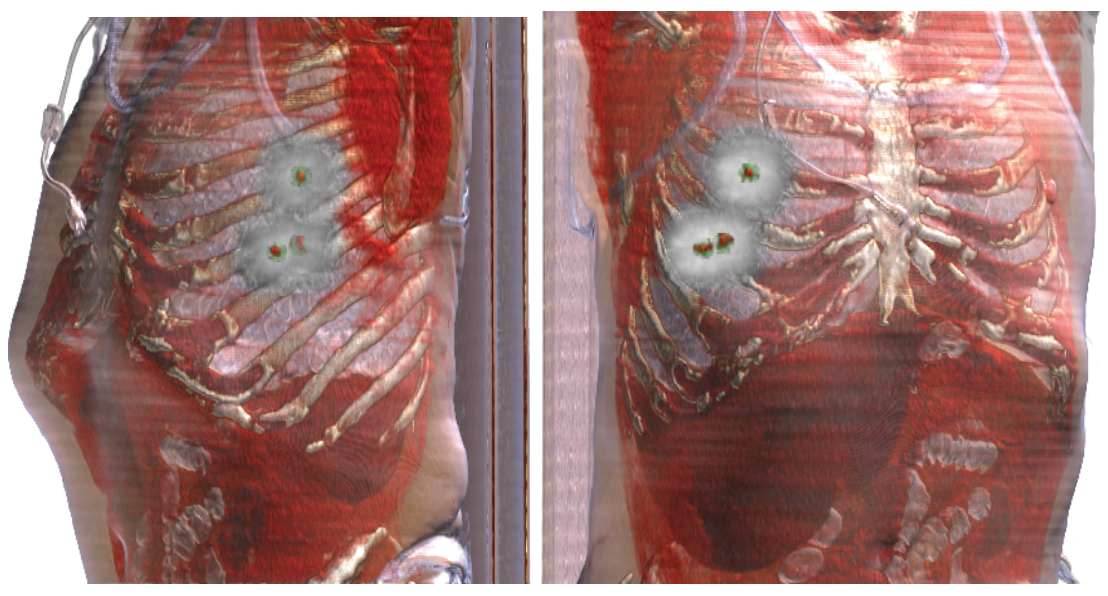

Fig. 4. Topology-driven visualizations of suspicious regions (possibly early-stage lung cancer nodules) within a thorax CT data. The suspicious regions are highlighted in red enclosed by a green halo [11].

\subsection{Visualization of Time-Varying Meteorological Data}

The second example where topology information controls the visualization pipeline is meteorological visualization. It is illustrated by visualizing the time-varying data of a hurricane (Isabel, 2003). The data consists of multiple variables including cloud density, precipitation, ice level, temperature, pressure, and velocity vector for each time-step. In this case the interesting feature is the vortex core, i.e., the eye of the hurricane which is a topological structure. For the hurricane analysis it might be useful to have a clear view on this feature and to select which variable to visualize. Therefore the cutaway technique can be effectively applied in this case as well. The example 
also shows multiple variables using topology-driven visualization. The focus object is defined as the group of voxels inside a cylinder around the hurricane eye. Inside the cylinder the total precipitation mixing ratio is visualized. Due to the cut-away view it is possible to have a clear view of this property close to the eye of the hurricane. Outside the cylinder is the context area where the total cloud moisture is visualized. In this time-dependent dataset the important feature changes its position and shape over time. Importance-driven visualization guarantees to emphasize the important feature irrespective of viewpoint and feature position and shape. A single time-step is shown in Figure 5.

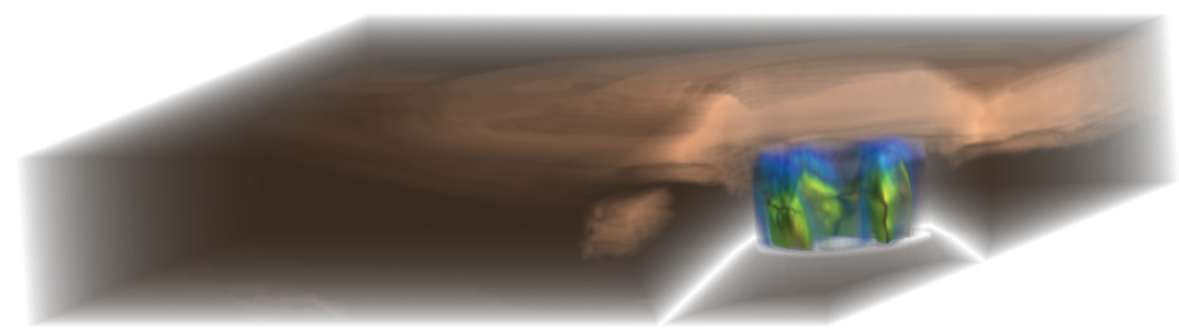

Fig. 5. Cut-away visualization of a multidimensional volumetric data of hurricane Isabel.

\section{Topology Changes of the Underlying Data}

The third type of visualizations enhances the visibility of interesting objects by (virtually) changing the topology of the underlying data. Examples include exploded views, a concept known from illustrations and assembly manuals, and other specific visualizations from computer-aided diagnosis.

\subsection{Exploded Views}

Exploded views modify the spatial arrangement of features to uncover the most prominent ones and thus change the topology of the underlying data. A multi-object visualization technique related to exploded views has been presented by Grimm et al. [1]. They present a data structure denoted as $V$-Objects for individual handling and manipulation of features within a volumetric dataset. An example of a topological change is shown in Figure 6. Here the context information is the skull which occludes the brain in focus. The topology of the skull is changed through the exploded views concept to unveil the focus. 


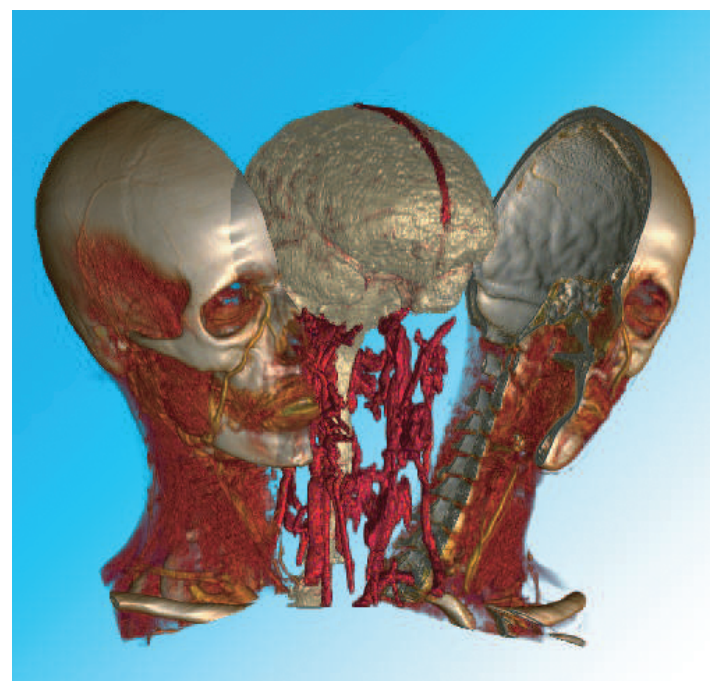

Fig. 6. Multi-volume visualization enabling exploded views through topology change of the underlying data [1].

\subsection{Colon Unfolding}

In this section we discuss virtual colon unfolding from medical visualization. The goal is to clearly present all relevant information in a single image. The approach performs unfolding of a cylindrical structure to a plane. This incurs a topological change of the original tubular structure. The unfolding is applied to computed tomography data of the colon. The traditional way of colon inspection for polyps (early-stage colon cancer) is using a real-world endoscope. Such an inspection process is uncomfortable for the patient. Virtual colonoscopy has been applied recently. The endoscope is replaced by a virtual endoscope traversing the tomographic data instead of the patient's body. This technique is much more comfortable, but the diagnosis is still time-consuming. Unfolding the tubular structure provides an instant overview of the entire organ [10]. In this case the topological change is important for a rapid diagnosis. An image of part of an unfolded colon with identified polyps is shown in Figure 7 .

\section{Conclusions}

In this paper we have discussed three scenarios where topology and visualization play together: visualization of topology, topology-driven visualization, and topology changes of the underlying data.

We have dealt with topology-based techniques for visualization and feature definition. The sparse visual representation showing topological characteris- 


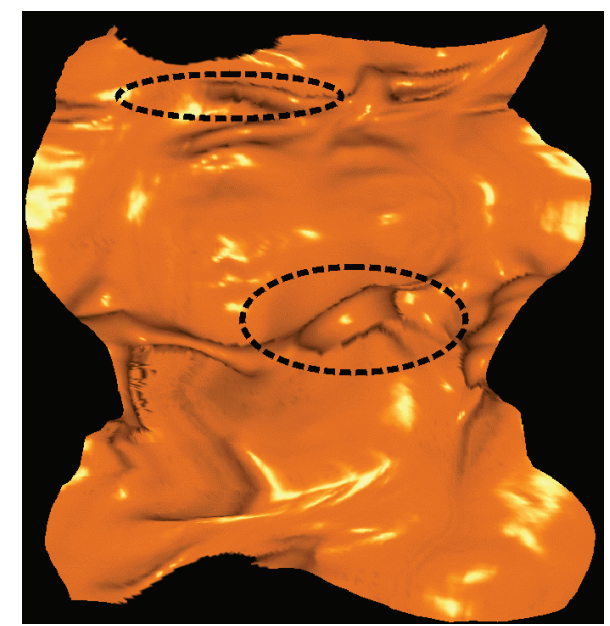

Fig. 7. Virtual colon unfolding using a topology change in the visualization [10].

tics is very useful for flow visualization and dynamical-systems visualization. Topological analysis can be used to define the importance of interesting objects and express their relevance in cut-away visualizations. Topological changes of the data are often useful to increase the visibility of the most relevant objects. Examples demonstrate the significance of topology information for a broad spectrum of expressive focus + context visualizations.

\section{Acknowledgments}

The authors thank Tino Weinkauf and Holger Theissel for providing a polygonal model of the topological information of the flow in Figure 1. The work presented in this publication is carried out as part of the exvisation project (www.cg.tuwien.ac.at/research/vis/exvisation) supported by the Austrian Science Fund (FWF) under grant no. P18322.

\section{References}

1. S. Grimm, S. Bruckner, A. Kanitsar, and E. Gröller. Flexible direct multivolume rendering in interactive scenes. In Proceedings of Vision, Modeling, and Visualization'04, pages 379-386, 2004.

2. H. Hauser. Scientific Visualization: The Visual Extraction of Knowledge from Data, chapter Generalizing Focus+Context Visualization, pages 305-327. Springer-Verlag, 2005.

3. J. Hladůvka. Derivatives and Eigensystems for Volume-Data Analysis and Visualization. PhD thesis, Vienna University of Technology, Austria, 2001. 
4. H. Löffelmann. Visualizing Local Properties and Characteristic Structures of Dynamical Systems. PhD thesis, Vienna University of Technology, Austria, 1998.

5. Y. Sato, C. Westin, A. Bhalerao, S. Nakajima, N. Shiraga, S. Yoshida, and R. Kikinis. Tissue classification based on $3 \mathrm{~d}$ local intensity structures for volume rendering. IEEE Transactions on Visualization and Computer Graphics, 6(2):160-180, 2000.

6. H. Theisel, T. Weinkauf, H.-C. Hege, and H.-P. Seidel. Saddle connectors - an approach to visualizing the topological skeleton of complex $3 \mathrm{D}$ vector fields. In Proceedings of IEEE Visualization 2003, pages 225-232, 2003.

7. H. Theisel, T. Weinkauf, H.-C. Hege, and H.-P. Seidel. Topological methods for $2 \mathrm{~d}$ time-dependent vector fields based on stream lines and path lines. IEEE Transactions on Visualization and Computer Graphics, 11(4):383-394, 2005.

8. X. Tricoche, C. Garth, G. Kindlmann, E. Deines, G. Scheuermann, M. Ruetten, and C. Hansen. Visualization of intricate flow structures for vortex breakdown analysis. In Proceedings of IEEE Visualization 2004, pages 187-194, 2004.

9. J. J. van Wijk. Spot noise: Texture synthesis for data visualization. Computer Graphics, 25(4):319-318, 1991.

10. A. Vilanova. Visualization Techniques for Virtual Endoscopy. PhD thesis, Vienna University of Technology, Austria, 2001.

11. I. Viola. Importance-Driven Expressive Visualization. PhD thesis, Vienna University of Technology, Austria, 2005.

12. I. Viola, A. Kanitsar, and M. E. Gröller. Importance-driven feature enhancement in volume visualization. IEEE Transactions on Visualization and Computer Graphics, 11(4):408-418, 2005.

13. T. Weinkauf, H. Theisel, H.-C. Hege, and H.-P. Seidel. Topological construction and visualization of higher order 3D vector fields. In Proceedings of Eurographics 2004, pages 469-478, 2004.

14. X. Zheng and A. Pang. Topological lines in 3D tensor fields. In Proceedings of IEEE Visualization 2004, pages 313-320, 2004. 